\title{
Size Effect for Barium Titanate Nano-particles ${ }^{\dagger}$
}

\author{
Tomoya Ohno, Daisuke Suzuki, \\ and Hisao Suzuki \\ Shizuoka University, Faculty of Engineering* \\ Takashi Ida \\ Nagoya Institute of Technology, Ceramic Research \\ Laboratory**
}

\begin{abstract}
Barium titanate (BT) nano-particles with different diameters were prepared by Sol-Gel method. The particle diameter was determined by XRD, and the lattice constants of nano-particles were also determined by XRD. We also measured the lattice vibration for BT nano-particles by raman scattering to discuss on the size effect. As a result, we successfully calculated the intrinsic dielectric constants for BT nano-particles by analyzing the lattice vibration. Moreover, we confirmed the shift of curie temperature by the size effect.
\end{abstract}

\section{Introduction}

Barium titanate (BT) is one of the most important dielectric materials for the electronic devices, such as MLCC (Multi Layer Ceramic Capacitor). The thickness of the barium titanate thin film in MLCC has become thinner and reached about $1 \mu \mathrm{m}$. Further down sizing is required for the higher performance. For this reason, we should take into account for the size effect of BT nano particles. The critical size for BT has been reported to be spread out in the wide range between 25 and $110 \mathrm{~nm}$, depending on the measurement technique [1], [2]. On the other hand, the change in the electrical properties by the size effect has not been reported.

In this study, we measured the critical particle diameter by XRD and raman scattering in detail to show the phase transition by the size effect. In our previous works, the intrinsic dielectric constants for a lead titanate and lead zirconate titanate nano-particles were calculated by analyzing the raman spectra [3], [4]. In this study, we tried to use the same technique

\footnotetext{
* 3-5-1 Johoku, Hamamatsu, Shizuoka 432-8561, Japan

**10-6-29, Asahigaoka, Tajimi, Gifu 507-0071

$\uparrow$ This report was originally printed in J. Soc. Powder Technology, Japan, 41(2), 86-91 (2004) in Japanese, before being translated into English by KONA Editorial Committee with the permission of the editorial committee of the Soc. Powder Technology, Japan.
}

to observe the dielectric behavior for BT nano-particles.

\section{Experimental Procedure}

Barium titanate nano-particles were prepared by Sol-Gel method. The starting reagents for barium titanate precursor solution are the metal barium and titanium iso-propoxide. At first, metal barium was refluxed in ethanol at $80^{\circ} \mathrm{C}$ for 1 hour to prepare the barium alkoxide solution. Next, titanium iso-propoxide was mixed in the barium alkoxide solution, and was refluxed at $80^{\circ} \mathrm{C}$ for 1 hour to prepare $\mathrm{BT}$ precursor solution. The BT precursor particle was prepared by evaporating the solvent from the precursor solution. The BT nano-particles with different diameter were prepared by calcining the BT precursor particle at $350^{\circ} \mathrm{C}$ to remove the residual organics followed by the annealing at different temperatures to deposit the BT nano-particles with different diameters.

The particle diameter was determined by XRD analysis by using Scherrer's equation. The phase transition by the size effect was observed by raman scattering, as well as the intrinsic dielectric constant. The shift of curie temperature was determined by raman analysis. The raman spectra were measured using JASCO Co., NR-1800, Rev.1.00 raman spectrometer in backscattering geometries. The $488 \mathrm{~nm}$ line of an argon ion laser was used as the excitation 
source at power level of $50 \mathrm{~mW}$. Each spectrum was

othe ressult of the addition of several scans.

\section{Results and Discussion}

\subsection{Change in lattice constant with particle diameter}

The particle diameter was measured by XRD analysis using deconvolution technique [5] because the lattice constants for BT nano-particles along a- and c-axes exhibited very near values. Thus the observed XRD patterns were fitted by the Lorenz function to determine the half band width and peak angle, giving rise to the precise calculation of the lattice constants and the particle diameters. The particle diameter was calculated by using Scherrer's equation (equation 1).

$$
D=\frac{K \lambda}{\beta \cos \theta}
$$

where, $\mathrm{K}(=0.9)$ is Scherrer's constant, $\lambda$ the wave length of X-ray and $\beta$ the FWHM.

Figure 1 shows the lattice constant dependence as a function of particle diameter. It was found from this figure that the tetragonality decreased abruptly with decreasing particle diameter at around $30 \mathrm{~nm}$, showing the abrupt phase transition of BT nano-particles compared with the phase transition of a lead titanate nano-particles [6]. In addition, the tetragonality of the $100 \mathrm{~nm}$ particle was considered to be lower than that of a single crystal at room temperature. Abrupt change in the lattice constants shown in Fig. 1 and/or the size effect is ascribed to the rapid overdumping of soft modes [7] and the size dependence in the lattice constants for BT nano-particles exhibited almost same behavior as that reported by Uchino et. al. [2].

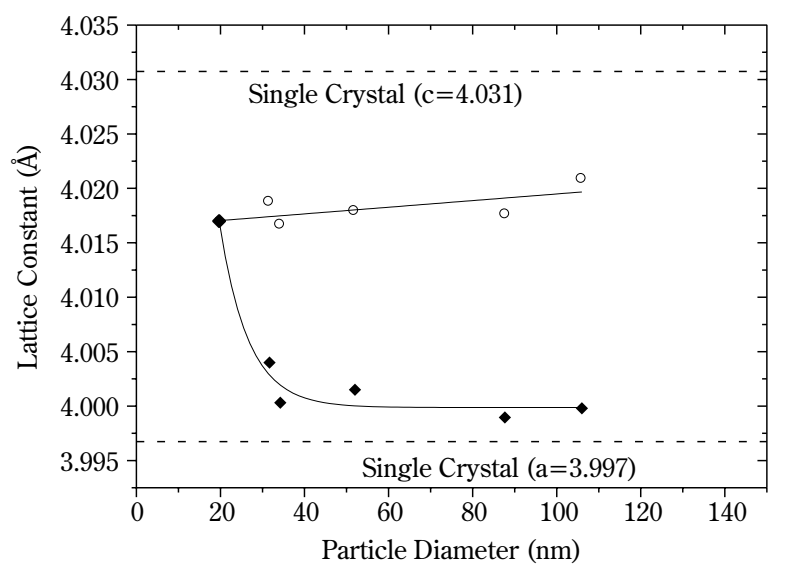

Fig. 1 The lattice constant with particle diameter

\subsection{Raman analysis}

In this study, accurate phase symmetry change with particle diameter or critical size was determined by the raman scattering because it was very difficult to determine the critical size for BT only by the XRD analysis due to the small difference in the lattice constants along a- and c-axes. By the raman scattering technique, it is easier to identify the crystal symmetry because tetragonal $\mathrm{BT}$ particle has the raman active crystal symmetry of $C_{4 v}^{1}$ to show the distinguishable raman peaks at ambient temperature in contrast to the cubic BT nano-particles of the raman inactive $\mathrm{O}_{\mathrm{h}}$ symmetry, leading to the peak disappearance at the critical particle diameter and critical temperature of curie point. Therefore, we can identify the crystal symmetry of BT nano-particles in principle by the existence of raman peaks at the measuring temperature. However, in the case of BT, it is reported that some phonon modes remain even for the cubic structure, because of the lattice relaxation and the secondary raman scattering [8]. Therefore in this study, raman spectrum of a $\mathrm{BT}$ single crystal was measured at above the curie temperature of $120^{\circ} \mathrm{C}$ to confirm the residual peak positions by the lattice relaxation and the secondary scattering. By comparing this raman spectrum of a BT single crystal measured at above the curie temperature and the measured raman spectra of the BT nano-particles, we can easily identify the crystal symmetry of BT nano-particles.

Figure 2 shows the raman spectra for the barium titanate nano-particles with different diameters at room temperature. The observed raman spectra were fitted by simply the sum of damped harmonic oscillators and a Debye relaxation mode. The lattice mode was assigned by analyzing the single crystal data. However, it was very difficult to determine the exact mode frequencies for BT because of their very small difference in the mode frequencies. Therefore, we used polarized laser to assign the accurate mode frequencies for a BT single crystal. Table 1 listed the index in space group for tetragonal BT. From Table 1, only A(LO) modes are detected in raman scattering under the condition of $\mathrm{X}(\mathrm{ZZ}) \mathrm{X}$ (enter the Zaxis polarized incident laser from the $\mathrm{X}$ plane of $\mathrm{BT}$ single crystal, and then measure the reflected scattering polarized along Z-axis; hereafter the measuring condition is denoted such like this). In this study, $\mathrm{A}(\mathrm{TO}), \mathrm{A}(\mathrm{LO})$ and $\mathrm{E}(\mathrm{LO})$ modes were measured under the conditions of $Z(Y Y) Z, X(Z Z) X$ and $X(Z Y) X$, respectively. Here, a sharp peak was obseved at $308 \mathrm{~cm}^{-1}$ when measured under the condition of $Z(Y X) Z$ to confirm a silent mode. The peak near 
200 cra 9 was confirmed by the measurements under the conditions of $\mathrm{X}(\mathrm{ZZ}) \mathrm{X}$ and $\mathrm{X}(\mathrm{ZY}) \mathrm{X}$ to be the superimpose of E(1LO) and A1(1LO) modes. Furthermore, it has been reported that the peak near $200 \mathrm{~cm}^{-1}$ was also observed by the raman analysis under the $\mathrm{X}(\mathrm{ZY}) \mathrm{Z}$ condition. Therefore, we assigned at last the peak near $200 \mathrm{~cm}^{-1}$ to be the superimpose of three peaks of $\mathrm{E}(1 \mathrm{LO}), \mathrm{A}(1 \mathrm{LO})$ and $\mathrm{E}(2 \mathrm{TO})$. The observed results in this study were in good agreement with those of the results for a BT single crystal by another reports.

Figure 3 shows the raman spectra for a BT single crystal using the polarized lazer under the different conditions. However, raman spectrum under the

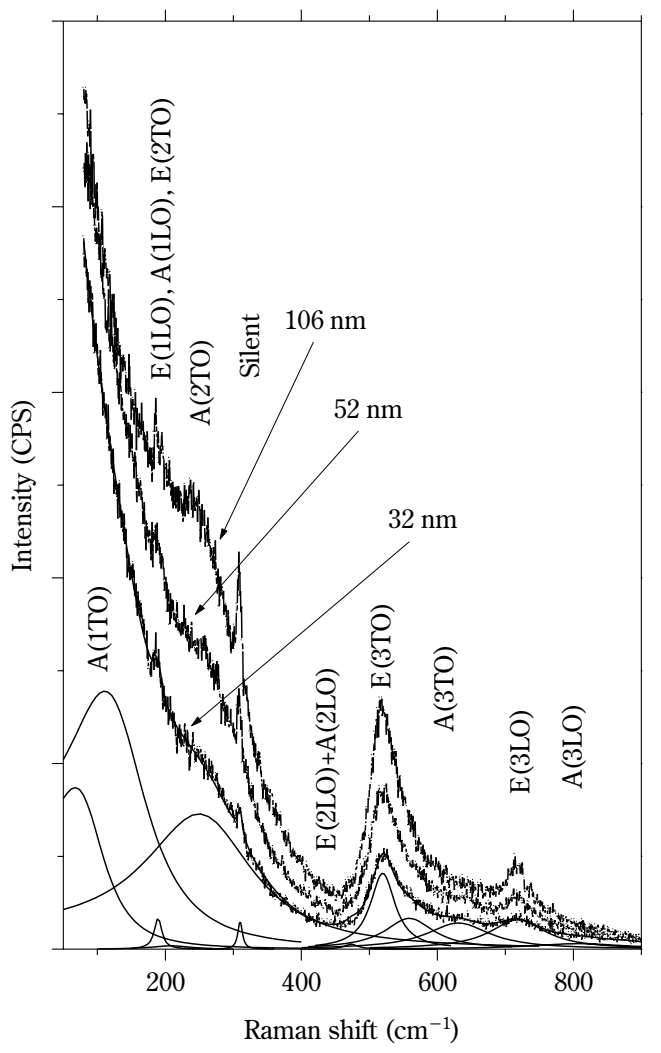

Fig. 2 Raman spectra for BT nano-particles with different particle diameters.

Table 1 Index for tetragonal system in space group

\begin{tabular}{cc}
\hline \multicolumn{3}{c}{ Space Group: $C_{4 v}^{1}$} \\
\hline $\mathrm{A}_{1}$ & $\alpha_{\mathrm{xx}}+\alpha_{\mathrm{yy}}, \alpha_{\mathrm{zz}}$ \\
$\mathrm{B}$ & $\alpha_{\mathrm{xy}}$ \\
$\mathrm{E}$ & $\left(\alpha_{\mathrm{yz}}, \alpha_{\mathrm{zx}}\right)$ \\
\hline
\end{tabular}

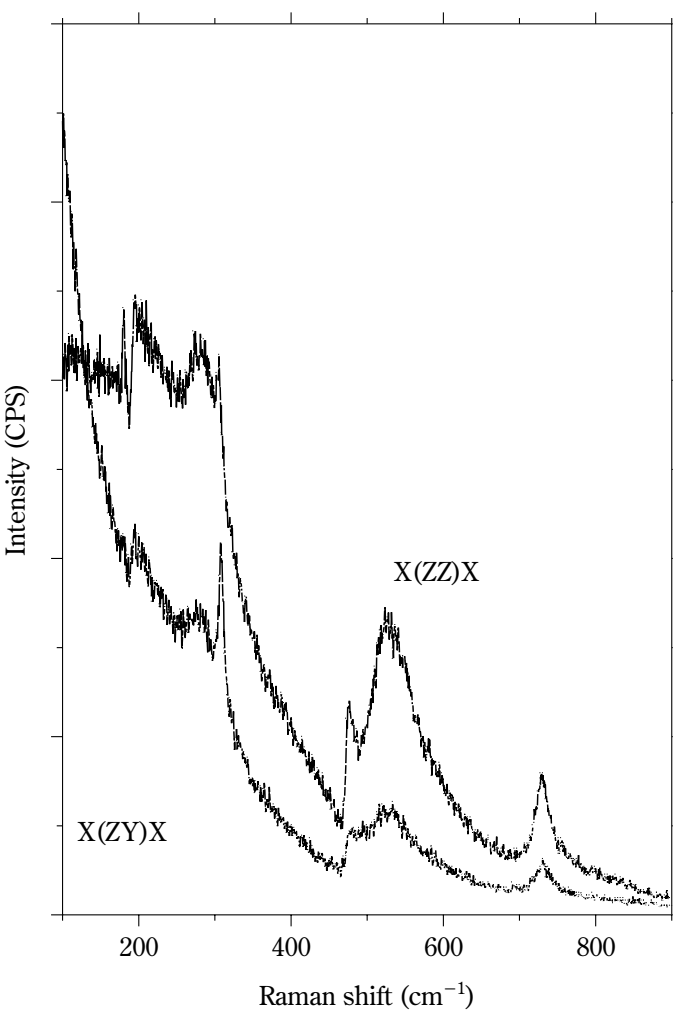

Fig. 3 (a) Raman spectra of BT single crystal (1)

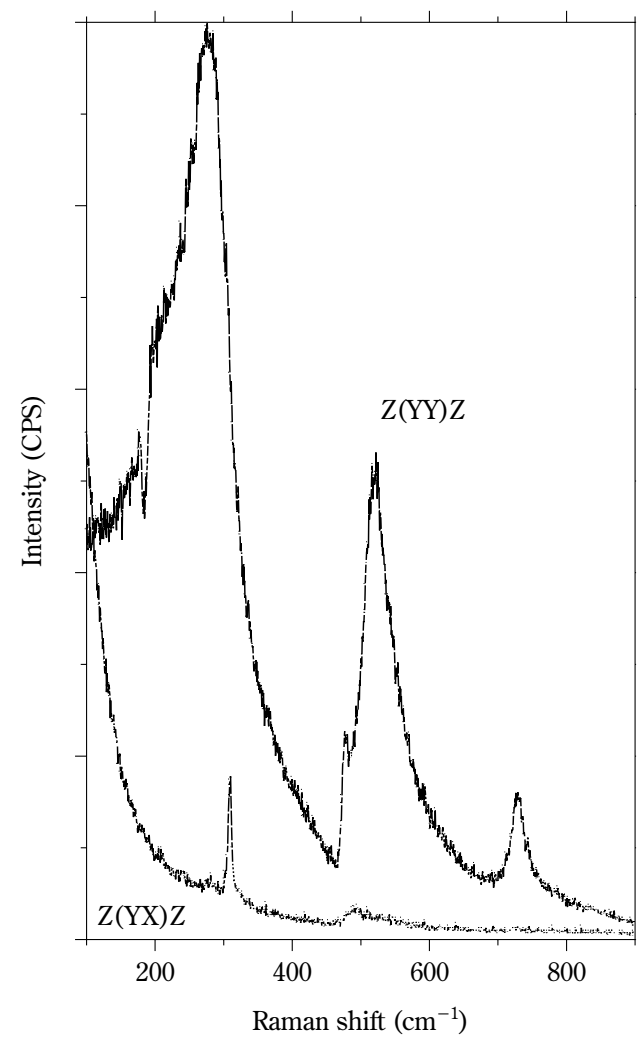

Fig. 3 (b) Raman spectra of BT single crystal (2) 
$\mathrm{X}(\mathrm{ZY}) \mathrm{Z}$ Gordition $\left(90^{\circ}\right.$ scattering) could not be observed in this measurement to assign only the E(TO) mode because of the sample limitation. Therefore, we used the reported value for E(TO) mode [7], [9] to analyze the raman spectra for a BT single crystal, leading to the accurate raman analysis for BT nanoparticles. These analyses enabled the calculation of the intrinsic dielectric constant for a BT single crystal by using the LST relation. As a result, the intrinsic dielectric constants of a BT single crystal along a- and c-axes were calculated to be 1247 and 44, respectively. These values were in good agreement with those reported for a BT single crystal $\left(\varepsilon_{\mathrm{a}}=1500, \varepsilon_{\mathrm{c}}=38\right)$ [7], [9], indicating the accuracy of the measurement technique.

\subsection{Change in curie temperature with particle diameter}

Generally, the size effect for ferroelectric materials results in the curie temperature shift to the lower temperature. Therefore, the curie temperature shift for BT nano-particles was investigated in this study. Figure 4 shows the curie temperature shift as a function of the particle diameter. As already pointed out, some lattice vibration remained above the curie temperature by the secondary scattering and so on, in the case of BT. Hence, the raman spectrum for a BT single crystal was measured at above curie temperature of $140^{\circ} \mathrm{C}$ to assign the residual peaks by the secondary scattering and so on. By this treatment, it was found that the curie temperature for BT nano-particles was shifted to the lower temperature at around 20-30 nm of particle diameter. This tendency was

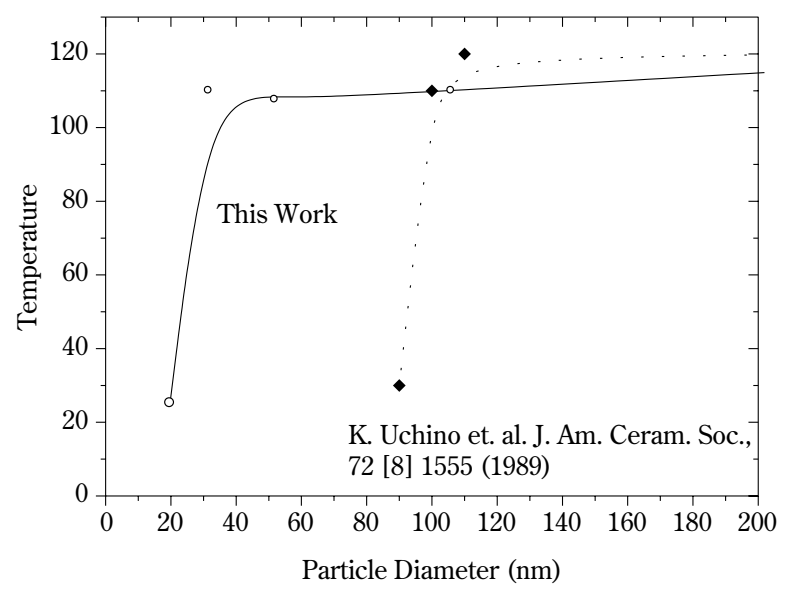

Fig. 4 Change in the curie temperature as a function of particle diameter almost same as that of the result of Uchino et. al. measured by the change in the lattice constants as a function of the particle diameter using XRD. However, their result showed that the curie temperature shift to the lower temperature began at around $100 \mathrm{~nm}$ of particle diameter. This difference in the critical size for BT was ascribes to the measurement techniques of the particle diameter and the crystal symmetry.

\subsection{Change in dielectric constant with particle diameter}

Whereas there have been many reports on the size effect for ferroelectric materials, there is no report about size effect on the electrical properties for ferroelectric nano-particles. This was ascribed to the difficulty in the measurement of the electrical properties for nano-particles. For example, in the case of the dielectric measurement, the impedance analyzer is used to measure the capacitance of the materials to calculate the dielectric constant. However, by this measurement technique, only the extrinsic dielectric constant including the effect of pores, space charge and so on was estimated, leading to the misunderstanding of the dielectric behavior in the case of the ferroelectric nano-particles which could not be compacted into the dense green body. The measurement of the extrinsic dielectric constant is useful to apply the ferroelectric materials to the electronic devices, however, the intrinsic dielectric constant could not be measured by this method. Therefore, in this research, the intrinsic dielectric constant, not including the effect of pores, space charge and so on, was estimated from the raman spectra by using the LST relation (equation 2 ).

$$
\frac{\varepsilon}{\varepsilon_{\infty}}=\frac{\omega_{1 L O}^{2}}{\omega_{1 T O}^{2}} * \frac{\omega_{2 L O}^{2}}{\omega_{2 T O}^{2}} * \frac{\omega_{3 L O}^{2}}{\omega_{3 T O}^{2}}
$$

where, $\omega$ is the mode frequency for each phonon modes, and $\varepsilon_{\infty}$ is optical dielectric constant $(\mathrm{a}=5.22$, $\mathrm{c}=5.07)$. Table 2 lists the observed mode frequencies for A mode (c-axis direction) of a BT nano-particle with $105 \mathrm{~nm}$. The mode frequencies were calculated by the fitting the spectrum using the damped harmonic oscillators [4], [8]. From these calculation, the intrinsic dielectric constant for BT nano-particle with

Table 2 Mode frequencies from the BT nano crystal with $105 \mathrm{~nm}$ diameter

\begin{tabular}{|c|c|c|c|c|c|}
\hline $\mathrm{A}(1 \mathrm{TO})$ & $\mathrm{A}(2 \mathrm{TO})$ & $\mathrm{A}(3 \mathrm{TO})$ & $\mathrm{A}(1 \mathrm{LO})$ & $\mathrm{A}(2 \mathrm{LO})$ & $\mathrm{A}(3 \mathrm{LO})$ \\
\hline $159.3 \mathrm{~cm}^{-1}$ & $262.0 \mathrm{~cm}^{-1}$ & $548.1 \mathrm{~cm}^{-1}$ & $193.3 \mathrm{~cm}^{-1}$ & $460.5 \mathrm{~cm}^{-1}$ & $739.5 \mathrm{~cm}^{-1}$ \\
\hline
\end{tabular}


105 nom diameter along c-axis was calculated to be fe 10 42.0. In addition, intrinsic dielectric constant for W'BT nano-particle with $105 \mathrm{~nm}$ diameter along a-axis was calculated to be $\varepsilon_{\mathrm{a}}=1254.6$ by the calculation for E modes.

In the previous section, the curie temperature was confirmed to shift to the lower temperature by the size effect. This suggests that the dielectric behavior should change according to that illustrated in Figure 5. Therefore, it is speculated that the intrinsic dielectric constant increases at room temperature by the size effect. In this study, the intrinsic dielectric constant of BT nano-particles calculated from the equation (2) increased with decreasing particle diameter at around $40 \mathrm{~nm}$ as shown in Figure 6. Further-

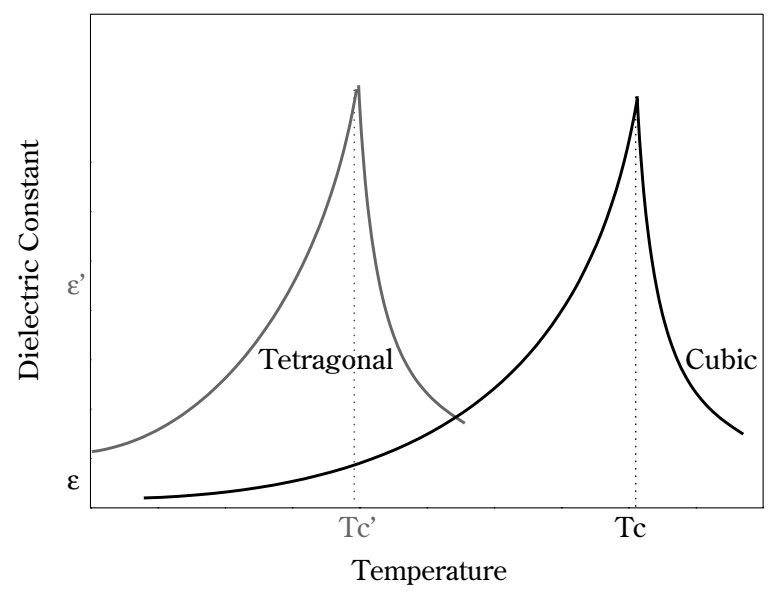

Fig. 5 Schematic illustration for the curie temperature shift of BT nanocrystals

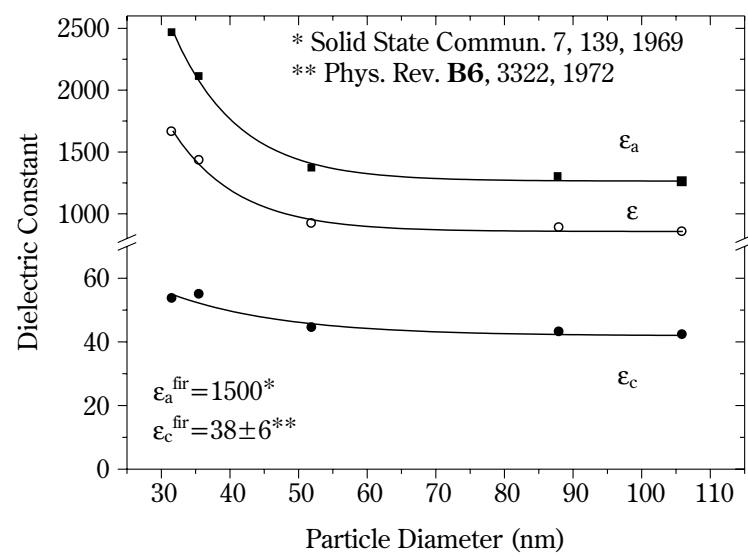

Fig. 6 Change in the intrinsic dielectric constant for BT nanocrystals estimated by raman scattering with particle diameter more, the intrinsic dielectric constant for a BT nanoparticle with $30 \mathrm{~nm}$ particle diameter exhibited a larger value than that for a single crystal. These results demonstrate that the intrinsic dielectric constant increases drastically with decreasing particle diameter according to the equation (2), which suggests the possibility for high $\mathrm{K}$ (dielectric) materials with thin layers of ferroelectric nano-particles.

\subsection{Evaluation of critical size for BT}

From the Figs. 1 and 4, the tetragonality for BT nano-particles decreased dramatically at around 40 $\mathrm{nm}$ of particle diameter, and finally the crystal structure changed to cubic phase at $20 \mathrm{~nm}$. In addition, the intrinsic dielectric constant started to increase at around $40-50 \mathrm{~nm}$, and reached larger value than that for a single crystal if the particle diameter was below $40 \mathrm{~nm}$. Furthermore, it was also confirmed from the raman analysis that the crystal structure was perfectly transformed to the cubic phase at $20 \mathrm{~nm}$ of particle diameter. Zhong et. al. has been reported that the onset of the surface relaxation was calculated to start at the size of $43 \mathrm{~nm}$ based on the surface relaxation model, in which the surface part of the particles were consisted of the cubic phase [10]. This theoretical consideration will sustain our experimental results. Therefore, we concluded that the critical size determined by the raman analysis together with the $\mathrm{XRD}$ analysis was reasonable and the raman analysis was the powerful tool for analyzing the ferroelectric materials.

\section{Conclusion}

In this study, we demonstrated that the size effect for BT nano-particles could be estimated by using the raman analysis together with the XRD analysis. As a result, the phase transition by the size effect for a BT was successfully confirmed to start at around 20-30 $\mathrm{nm}$ of particle diameter. Therefore, the critical size of BT was considered to be about $20 \mathrm{~nm}$. On the other hand, we succeed to separate the exact phonon mode frequencies in raman spectra of BT nano-particles by measuring the fundamental mode frequencies for a single crystal. In addition, the intrinsic dielectric constants for BT nano-particles were successfully calculated by substituting the exact phonon mode frequencies for a LST relation. These results successfully demonstrated that the raman scattering is the powerful tool not only for the analysis of the size effect but also the measurement of the intrinsic dielectric behavior of ferroelectric materials. 
[6] Ishikawa, K., K. Yoshikawa and N. Okada: "Size effect on the ferroelectric phase transition in $\mathrm{PbTiO}_{3}$ ultrafine particles” Phys. Rev. B: Condens. Matter. 37, 5852 (1988)

[7] Scalabrin, A., A. S. Chaves, D. S. Shim and S. P. S. Porto: "Temperature Dependence of the $\mathrm{A}_{1}$ and E optical phonons in $\mathrm{BaTiO}_{3}$ ” Phys. Stat. Sol. (b), 79, 731 (1977)

[8] Fu, D. S., H. Iwazaki, H. Suzuki and K. Ishikawa: "Phonon mode behaviors of $\mathrm{PbTiO}_{3}$ thin films deposited on Pt/Si substrates” J. Phys.: Condens. Matter., 12, 399 (2000)

[9] Domenico, M., S. H. Wemple, S. P. S. Porto and R. P. Bauman: "Raman Spectrum of Single-Domain $\mathrm{BaTiO}_{3}$ " Phys. Rev. 174, 522 (1968)

[10] Zhong, W. L., Y. G. Wang, P. L. Zang and B. D. Qu: "Phenomenological study of the size effect on phase transitions in ferroelectric particles” Phys. Rev. B50, 698 (1994) 


\section{Author's short biography}

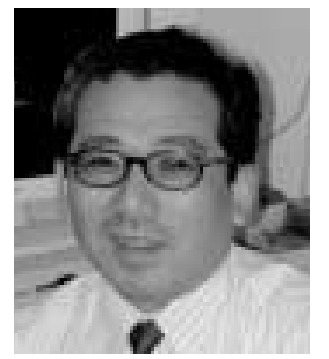

\section{Hisao Suzuki}

Prof. Hisao Suzuki has been at Department of Materials Science, Shizuoka University, Japan since 1994. He was also a Prof. at Mechanical System Engineering of Toyota Technological Institute from 1982 to 1994. He is now one of the advisory committee of the Society of Sol-Gel Science and the Society of Powder Technology and was one of the editorial board of the Journal of the Powder Technology, Japan and Journal of Advanced Powder Technology. He was also one of the editorial board of the Bulletin of Ceramics. His major research areas lie in the chemical processing of advanced nano-materials and thin films to apply the nano-devices, especially through the alkoxide route. His current activities are focused on the preparation of hybrid nano-particles by the nano-coating and hybrid integration of ferroelectric thin films with electrode and other nano-layers with controlled nanostructures for Micro(nano)-Electro-Mechanical Systems.

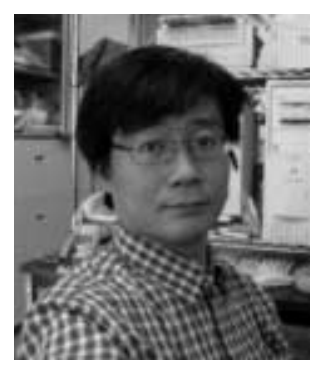

\section{Takashi Ida}

Prof. Takashi Ida has been at Ceramic Research Laboratory, Nagoya Institute of Technology, Japan since 1999. He was also a Research Associate at Department of Science, Himeji Institute of Technology from 1991 to 1999 and at Institute for Molecular Science from 1989 to 1991. His major research area lies in the development of a novel analytical method of X-ray diffraction. His current activities are focused on the analyses of the inorganic materials and powders.

\section{Tomoya Ohno}

Dr. Tomoya Ohno has been working at the innovative joint research center in Shizuoka University since 2004, as Post Doctoral Fellow under the direction of Prof. M. Fujimoto. He received Ph.D from Shizuoka University in 2004. His main research area lies in the raman analysis for ferroelectric nano-particles and thin films, as well as the electrophoresis control of DNA using nano-spheres.

\section{Daisuke Suzuki}

Daisuke Suzuki received his BE degree from the Department of Material Science at Shizuoka University in 2003 under direction of Prof. H. Suzuki. His main research area lies in the size effect for ferroelectric nano-particles. 